Marius Crainic · Ieke Moerdijk

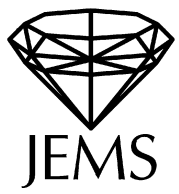

\title{
Deformations of Lie brackets: cohomological aspects
}

Received August 12, 2005 and in revised form July 8, 2007

Abstract. We introduce a new cohomology for Lie algebroids, and prove that it provides a differ-
ential graded Lie algebra which "controls" deformations of the structure bracket of the algebroid.

Keywords. Lie algebroids, deformations, cohomology, Poisson geometry

\section{Introduction}

The aim of this paper is to find the differential graded Lie algebra and its cohomology theory controlling deformations of a large class of geometric structures, known as Lie algebroids. This problem is particularly difficult since there is no "adjoint representation" to which one can apply the standard theory.

We recall that a Lie algebroid over a manifold $M$ is a vector bundle $\pi: A \rightarrow M$ together with a Lie bracket [, ] on the space of sections $\Gamma(A)$ and a bundle map $\rho$ : $A \rightarrow T M$, called the anchor, satisfying the Leibniz identity:

$$
[\alpha, f \beta]=f[\alpha, \beta]+L_{\rho \alpha}(f) \beta, \quad f \in C^{\infty}(M), \alpha, \beta \in \Gamma(A) .
$$

The notion of Lie algebroid in this form goes back to Pradines (1967), but, in local coordinates, it already appeared in E. Cartan's work in 1904, and the analogous algebraic notion was already studied by Rinehart in 1963.

Lie algebroids are to be thought of as infinite-dimensional Lie algebras of "geometric type", or as generalized tangent bundles. Indeed, the simplest examples are (finitedimensional) Lie algebras and tangent bundles of manifolds, and there are many natural geometric examples coming from foliations, Poisson manifolds, infinitesimal actions of Lie algebras on manifolds, and other contexts. The present paper is thus part of a larger programme, the goal of which is to give a unified approach to geometric structures controlled by "Lie brackets", and to make explicit the analogies and interplay between the various fields such as Lie group theory, theory of foliations, Poisson geometry, etc.

M. Crainic, I. Moerdijk: Department of Mathematics, Utrecht University, 3508 TA Utrecht, The Netherlands; e-mail: crainic@ math.uu.nl, moerdijk@ math.uu.nl

Mathematics Subject Classification (2000): Primary 58H05, 58H15; Secondary 57R30, 53D17 
A well-known principle in mathematics, present already in the study of deformations of complex structures (cf. [8] and the references therein) and in the work of NijenhuisRichardson [17], and emphasized by P. Deligne, M. Kontsevich, and others, states that the deformation theory of a specific (type of) structure is governed by a naturally associated differential graded Lie algebra (dgla). Moreover, this dgla, or the graded Lie algebra given by its cohomology, should act on invariants associated to the structure. In this paper, we will exhibit for any Lie algebroid $A$ the dgla governing its deformations, the cohomology Lie algebra of which we call the deformation cohomology of $A$ and denote by $H_{\text {def }}^{*}(A)$. We will relate this cohomology to known cohomologies of $A$, and in particular prove that it acts on the "de Rham" cohomology of $A$. We will prove that deformations of the bracket of a given algebroid $A$ give rise to cohomology classes in $H_{\text {def }}^{2}(A)$, and we investigate the relation of these classes to those constructed earlier for the deformation of foliations [10] and of Poisson brackets. We will also prove that, quite surprisingly, the vanishing of such cohomology classes does imply rigidity (Theorem 2).

In the extreme case where the Lie algebroid $A$ is simply a finite-dimensional Lie algebra, it is of course well-known that the deformations of the Lie bracket are controlled by (the cohomology of) the Chevalley-Eilenberg complex for the adjoint representation of $A$ [17, 18]. Our theory contains this fact as a special case, and can be interpreted as a way of defining the cohomology of a general Lie algebroid with coefficients in its adjoint representation, in spite of the fact that the "adjoint representation" is not defined as a representation in the usual sense [5].

Based on the analogy with the adjoint representation and the rigidity properties of compact Lie groups, we state at the end of this paper a general "rigidity conjecture" for algebroids of compact type which we expect to have applications to the linearization problem [23] and other rigidity problems. Moreover, we prove this conjecture in two special cases, namely when the Lie algebroid is regular and when the Lie algebroid is defined from an infinitesimal action of a Lie algebra on a manifold. The first case is closely related to Weinstein's linearization theorem in the regular case [24]—note in particular that his proof uses the same techniques, based on a Bochner-type averaging and the van Est isomorphism for groupoids [3]. The second case is related to the linearization theorem for Poisson manifolds [1]. We also give an idea of a proof in the general case, based on a "lin"-version of the category of smooth manifolds, which we expect to be of independent interest.

\section{Multiderivations and the deformation complex}

The deformation complex of a Lie algebroid $A$ is defined as the complex $\left(C_{\mathrm{def}}^{*}(A), \delta\right)$ in which the $n$-cochains $D \in C_{\mathrm{def}}^{n}(A)$ are $\mathbb{R}$-multilinear antisymmetric maps

$$
D: \underbrace{\Gamma(A) \times \cdots \times \Gamma(A)}_{n \text { times }} \rightarrow \Gamma(A)
$$


which are multiderivations, and the coboundary is given by

$$
\begin{aligned}
\delta(D)\left(\alpha_{0}, \ldots, \alpha_{n}\right)= & \sum_{i}(-1)^{i}\left[\alpha_{i}, D\left(\alpha_{0}, \ldots, \hat{\alpha_{i}}, \ldots, \alpha_{n}\right)\right] \\
& +\sum_{i<j}(-1)^{i+j} D\left(\left[\alpha_{i}, \alpha_{j}\right], \alpha_{0}, \ldots, \hat{\alpha}_{i}, \ldots, \hat{\alpha}_{j}, \ldots, \alpha_{n}\right) .
\end{aligned}
$$

We denote by $H_{\mathrm{def}}^{*}(A)$ the resulting cohomology. In this section we explain this definition and we investigate the structure present on $\left(C_{\mathrm{def}}^{*}(A), \delta\right)$.

\subsection{Multiderivations}

Let $E \rightarrow M$ be a vector bundle, and denote by $r$ its rank. Recall that a derivation on $E$ is any linear operator $D: \Gamma(E) \rightarrow \Gamma(E)$ with the property that there exists a vector field $\sigma_{D} \in \mathcal{X}(M)$, called the symbol of $D$, such that

$$
D(f s)=f D(s)+\sigma_{D}(f) s
$$

for any section $s \in \Gamma(E)$ and function $f \in C^{\infty}(M)$. We denote by $\operatorname{Der}(E)$ the space of derivations on $E$.

Assume, for the moment, that the rank of $E$ is $r \geq 2$. A multiderivation of degree $n$ is a skew-symmetric multilinear map

$$
D: \underbrace{\Gamma(E) \otimes \cdots \otimes \Gamma(E)}_{n+1 \text { times }} \rightarrow \Gamma(E)
$$

which is a derivation in each entry, i.e., there is a map

$$
\sigma_{D}: \underbrace{\Gamma(E) \otimes \cdots \otimes \Gamma(E)}_{n \text { times }} \rightarrow \mathcal{X}(M),
$$

called the symbol of $D$, such that

$$
D\left(s_{0}, s_{1}, \ldots, f s_{n}\right)=f D\left(s_{0}, s_{1}, \ldots, s_{n}\right)+\sigma_{D}\left(s_{0}, \ldots, s_{n-1}\right)(f) s_{n}
$$

for any function $f \in C^{\infty}(M)$ and sections $s_{i} \in \Gamma(E)$. Notice that this identity determines $\sigma_{D}$ uniquely.

We will denote by $\operatorname{Der}^{n}(E)$ the space of multiderivations of degree $n, n \geq 0$. We have $\operatorname{Der}^{0}(E)=\operatorname{Der}(E)$, and we set $\operatorname{Der}^{-1}(E)=\Gamma(E)$.

Lemma 1. For any multiderivation $D \in \operatorname{Der}^{n}(E)$ of degree $n \geq 0$, its symbol $\sigma_{D}$ is antisymmetric and $C^{\infty}(M)$-linear. Moreover, $\operatorname{Der}^{n}(E)$ is the space of sections of a vector bundle $\mathcal{D}^{n} E \rightarrow M$ which fits into a short exact sequence of vector bundles

$$
0 \rightarrow \bigwedge^{n+1} E^{\vee} \otimes E \rightarrow \mathcal{D}^{n} E \rightarrow \bigwedge^{n} E^{\vee} \otimes T M \rightarrow 0,
$$

where $E^{\vee}$ denotes the dual of $E$. In particular, $\operatorname{Der}^{n}(E)=0$ for $n \geq \operatorname{rk}(E)+1$. 
Proof. The antisymmetry of $\sigma_{D}$ follows from that of $D$. From this it also follows that

$$
\begin{aligned}
D\left(s_{0}, s_{1}, \ldots, s_{i-1},\right. & \left.f s_{i}, s_{i+1}, \ldots, s_{n}\right) \\
& =f D\left(s_{0}, s_{1}, \ldots, s_{n}\right)+(-1)^{n-i} \sigma_{D}\left(s_{0}, \ldots, \hat{s}_{i}, \ldots, s_{n}\right)(f) s_{i} .
\end{aligned}
$$

Now compute $D\left(f s_{0}, g s_{1}, \ldots, s_{n}\right)$ in two ways: first by taking $f$ out followed by taking out $g$, and then the other way around. We obtain

$$
\begin{aligned}
\left(\sigma_{D}\left(f s_{0}, s_{2}, \ldots, s_{n}\right)\right. & \left.(g)-f \sigma_{D}\left(s_{0}, s_{2}, \ldots, s_{n}\right)(g)\right) s_{1} \\
& +\left(\sigma_{D}\left(g s_{1}, s_{2}, \ldots, s_{n}\right)(f)-g \sigma_{D}\left(s_{1}, s_{2}, \ldots, s_{n}\right)(f)\right) s_{0}=0 .
\end{aligned}
$$

Since $E$ was assumed to be of rank $r \geq 2$, it follows that

$$
\sigma_{D}\left(f s_{0}, s_{2}, \ldots, s_{n}\right)-f \sigma_{D}\left(s_{0}, s_{2}, \ldots, s_{n}\right)=0,
$$

i.e., $\sigma_{D}$ is $C^{\infty}(M)$-linear.

Observe now that, if $\nabla$ is a connection on $E$, then the operator

$$
L_{D}\left(s_{0}, \ldots, s_{n}\right)=D\left(s_{0}, \ldots, s_{n}\right)+(-1)^{n} \sum_{i}(-1)^{i+1} \nabla_{\sigma_{D}\left(s_{0}, \ldots, \hat{s}_{i}, \ldots, s_{n}\right)}\left(s_{i}\right)
$$

is antisymmetric and $C^{\infty}(M)$-multilinear. This shows that any connection $\nabla$ on $E$ determines an isomorphism of $C^{\infty}(M)$-modules

$$
\operatorname{Der}^{n}(E) \cong \Gamma\left(\bigwedge^{n+1} E^{\vee} \otimes E\right) \oplus \Gamma\left(\bigwedge^{n} E^{\vee} \otimes T M\right),
$$

which sends $D$ to $\left(L_{D}, \sigma_{D}\right)$. This proves the statement about the existence of a vector bundle $\mathcal{D}^{n} E$, and it shows at the same time that the choice of a connection $\nabla$ induces a splitting of the short exact sequence above.

A similar definition when the rank of $E$ is $r=1$ would not imply the $C^{\infty}(M)$-linearity of the symbols. To extend the definition of $\operatorname{Der}^{n}(E)$ to this case, we must require $C^{\infty}(M)$ linearity of its symbols.

\subsection{Multiderivations and brackets}

There is a close connection between the spaces $\operatorname{Der}^{n}(E)$ and Lie algebroids. First of all, the obvious bracket $[\cdot, \cdot]$ on $\operatorname{Der}(E)$ extends to $\operatorname{Der}^{*}(E)$ as follows:

Proposition 1. For $D_{1} \in \operatorname{Der}^{p}(E)$ and $D_{2} \in \operatorname{Der}^{q}(E)$ we define the Gerstenhaber bracket

$$
\left[D_{1}, D_{2}\right]=(-1)^{p q} D_{1} \circ D_{2}-D_{2} \circ D_{1},
$$

where

$$
\begin{aligned}
& D_{2} \circ D_{1}\left(s_{0}, \ldots, s_{p+q}\right) \\
& \left.\quad=\sum_{\tau}(-1)^{|\tau|} D_{2}\left(D_{1}\left(s_{\tau(0)}, \ldots, s_{\tau(p)}\right), s_{\tau(p+1)}, \ldots, s_{\tau(p+q)}\right)\right)
\end{aligned}
$$

and the sum is over all $(p+1, q)$-shuffles. Then $\left[D_{1}, D_{2}\right] \in \operatorname{Der}^{p+q}(E)$, and the resulting bracket $[\cdot, \cdot]$ makes Der* $(E)$ into a graded Lie algebra. 
Proof. It is well-known that the bracket above provides a gla structure on the space of skew-symmetric multilinear maps $\Gamma(E) \otimes \cdots \otimes \Gamma(E) \rightarrow \Gamma(E)$ [18]. Therefore, it suffices to show that the space of multiderivations is closed under this bracket, and this follows by a careful (but straightforward) calculation. The final conclusion of the computation is that $\left[D_{1}, D_{2}\right]$ is a multiderivation with symbol

$$
\sigma_{\left[D_{1}, D_{2}\right]}=\left((-1)^{p q} \sigma_{D_{1}} \circ D_{2}-\sigma_{D_{2}} \circ D_{1}\right)+\left[\sigma_{D_{1}}, \sigma_{D_{2}}\right],
$$

where $\sigma_{D_{1}} \circ D_{2}$ is given by the same formula as (2) above (so $\sigma_{D_{1}} \circ D_{2}=0$ if $p=0$ ), while

$$
\begin{aligned}
{\left[\sigma_{D_{1}}, \sigma_{D_{2}}\right]\left(s_{1}, \ldots,\right.} & \left.s_{p+q}\right) \\
& =\sum_{\tau}(-1)^{|\tau|}\left[\sigma_{D_{1}}\left(s_{\tau(1)}, \ldots, s_{\tau(p)}\right), \sigma_{D_{2}}\left(s_{\tau(p+1)}, \ldots, s_{\tau(p+q)}\right)\right]
\end{aligned}
$$

(sum over shuffles again).

The formulas in Proposition 1 are quite standard and go back to Gerstenhaber [7] (the case of algebras) and Nijenhuis and Richardson [18, 19] (the case of Lie algebras). Note, however, that we have chosen the signs differently, so as to match the classical formulas for Lie derivatives and the de Rham differential.

The gla structure in $\operatorname{Der}^{*}(E)$ allows us to give the following folklore description of Lie algebroids (going back at least to [7]):

Lemma 2. If $A$ is a vector bundle over $M$, then there exists a one-to-one correspondence between Lie algebroid structures on A and elements $m \in \operatorname{Der}^{1}(A)$ satisfying $[m, m]=0$.

The more familiar form of the definition of a Lie algebroid is obtained by letting $[\alpha, \beta]=$ $m(\alpha, \beta)$ (the Lie bracket) and $\rho=\sigma_{m}: A \rightarrow T M$ (the anchor).

The vector bundle $\mathcal{D}^{0} E$ itself is a Lie algebroid for any vector bundle $E$. The bracket is the one mentioned above (given by the commutators of derivations), while the anchor is just taking the symbol, $\rho(D)=\sigma_{D}$.

Let us recall [13] that a representation of a Lie algebroid $A \rightarrow M$ is a vector bundle $E \rightarrow M$ together with a flat $A$-connection $\nabla$ on $E$. This means that $\nabla: \Gamma(A) \otimes \Gamma(E) \rightarrow$ $\Gamma(E)$ is a bilinear map, written $(\alpha, s) \mapsto \nabla_{\alpha}(s)$, which satisfies the connection properties

$$
\nabla_{f \alpha}(s)=f \nabla_{\alpha}(s), \quad \nabla_{\alpha}(f s)=f \nabla_{\alpha}(s)+\rho \alpha(f) s,
$$

as well as the flatness condition

$$
\nabla_{[\alpha, \beta]}=\left[\nabla_{\alpha}, \nabla_{\beta}\right]
$$

We observe the following well-known fact:

Lemma 3. Given a Lie algebroid A over $M$, there exists a one-to-one correspondence between representations of $A$ and vector bundles $E$ over $M$ together with a Lie algebroid map $\nabla: A \rightarrow \mathcal{D}^{0} E$. 
The lemma suggests using the notation $\mathfrak{g l}(E)$ for the Lie algebroid $\mathcal{D}^{0} E$. Of course, $E$ is a representation of $\mathfrak{g l}(E)$, with the tautological action

$$
\nabla_{D}(s)=D(s)
$$

for $D \in D^{0}(E)$ and $s \in \Gamma(E)$. We point out that the Lie algebroid $\mathfrak{g l}(E)$ is always integrable: one checks easily that the Lie groupoid $G L(E) \rightrightarrows M$, for which the arrows $x \stackrel{g}{\rightarrow} y$ are the linear isomorphisms $g: E_{x} \rightarrow E_{y}$, has Lie algebroid precisely $\mathfrak{g l}(E)$ (see also [13]).

\subsection{Cohomology}

Recall that, given a representation $E=(E, \nabla)$ of a Lie algebroid $A$, the de Rham cohomology of $A$ with coefficients in $E$ [13], denoted $H^{*}(A ; E)$, is defined as the cohomology of the complex $\left(C^{*}(A ; E), \delta_{A, E}\right)$, where $C^{p}(A ; E)=\Gamma\left(\bigwedge^{p} A^{\vee} \otimes E\right)$ consists of $C^{\infty}(M)$-multilinear antisymmetric maps

$$
\underbrace{\Gamma(A) \times \cdots \times \Gamma(A)}_{p \text { times }} \ni\left(\alpha_{1}, \ldots, \alpha_{p}\right) \mapsto \omega\left(\alpha_{1}, \ldots, \alpha_{p}\right) \in \Gamma(E),
$$

with the differential $\delta_{A, E}: C^{p}(A ; E) \rightarrow C^{p+1}(A ; E)$ given by the usual ChevalleyEilenberg formula:

$$
\begin{aligned}
\delta_{A, E}(\omega)\left(\alpha_{1}, \ldots, \alpha_{p+1}\right)= & \sum_{i<j}(-1)^{i+j} \omega\left(\left[\alpha_{i}, \alpha_{j}\right], \alpha_{1}, \ldots, \hat{\alpha}_{i}, \ldots, \hat{\alpha}_{j}, \ldots, \alpha_{p+1}\right) \\
& +\sum_{i=1}^{p+1}(-1)^{i+1} \nabla_{\alpha_{i}}\left(\omega\left(\alpha_{1}, \ldots, \hat{\alpha}_{i}, \ldots, \alpha_{p+1}\right)\right) .
\end{aligned}
$$

When $E$ is the trivial line bundle (with $\nabla_{\alpha}=L_{\rho(\alpha)}$, the Lie derivative along $\rho(\alpha)$ ), we omit $E$ from the notation. In particular, the differential on $C^{*}(A)$ will be denoted $\delta_{A}$.

For instance, if $A=T M$ is the tangent bundle of $M$, then $C^{*}(A)=\Omega^{*}(M)$ and the formula for $\delta_{A}$ becomes the known Koszul formula for the de Rham differential. Also, when $A=\mathfrak{g}$ is a Lie algebra (and $M$ consists of one point), one recovers the ChevalleyEilenberg complex computing Lie algebra cohomology with coefficients. In general, if $E$ is a representation of the Lie algebroid $A$, then $\Gamma(E)$ becomes a representation of the Lie algebra $\Gamma(A)$, while the Lie algebroid complex

$$
C^{*}(A ; E) \subset C^{*}(\Gamma(A) ; \Gamma(E))
$$

is the subcomplex of $C^{*}(\Gamma(A) ; \Gamma(E))$ consisting of the $C^{\infty}(M)$-multilinear cochains.

\subsection{Deformation cohomology}

It is well-known that deformations of a Lie algebra $\mathfrak{g}$ are controlled by $H^{*}(\mathfrak{g}, \mathfrak{g})$, the cohomology of $\mathfrak{g}$ with coefficients in the adjoint representation, and by the associated differential graded Lie algebra $\left(C^{*}(\mathfrak{g} ; \mathfrak{g}), \delta\right)[18,19]$. Here, the graded Lie algebra structure 
is the one of Proposition 1 applied to the case where $M$ is a point and $E=\mathfrak{g}$, and the differential is the Chevalley-Eilenberg differential, which can also be expressed in terms of the Gerstenhaber bracket with the Lie bracket $m \in C^{2}(\mathfrak{g}, \mathfrak{g})$ of $\mathfrak{g}[18]$,

$$
\delta(c)=[m, c] .
$$

For the case of Lie algebroids, one faces the problem that, in general, Lie algebroids do not have an adjoint representation in the classical sense mentioned above (see also the discussion in [5]), and/or the $C^{\infty}(M)$-multilinear cochains of the complex $C^{*}(\Gamma(A) ; \Gamma(A))$ do not form a subcomplex. However, there is a distinguished subcomplex of $C^{*}(\Gamma(A) ; \Gamma(A))$ which consists of cocycles which are "not far from being $C^{\infty}(M)$-multilinear". More precisely, since the symbol of an element $D \in \operatorname{Der}^{*}(A)$ is uniquely determined by $D$, the multiderivations form a subcomplex

$$
\operatorname{Der}^{*-1}(A) \subset C^{*}(\Gamma(A) ; \Gamma(A)) .
$$

This is precisely the complex that we have denoted $C_{\mathrm{def}}^{*}(A)$ and called the deformation complex of $A$ at the beginning of this section. That this is indeed a subcomplex follows from Proposition 1 and the fact that $\delta=[m,-]$. It can also be shown directly that, for any multiderivation $D \in \operatorname{Der}^{n}(A), \delta(D)$ is again a multiderivation with the symbol

$$
\sigma_{\delta(D)}=\delta\left(\sigma_{D}\right)+(-1)^{n} \rho \circ D
$$

where, for $\sigma \in \Gamma\left(\bigwedge^{n} A^{\vee} \otimes T M\right), \delta(\sigma) \in \Gamma\left(\bigwedge^{n+1} A^{\vee} \otimes T M\right)$ is given by

$$
\begin{aligned}
\delta(\sigma)\left(\alpha_{0}, \ldots, \alpha_{n}\right)= & \sum_{i}(-1)^{i}\left[\rho\left(\alpha_{i}\right), \sigma\left(\alpha_{0}, \ldots, \hat{\alpha}_{i}, \ldots, \alpha_{n}\right)\right] \\
& +\sum_{i<j}(-1)^{i+j} \sigma\left(\left[\alpha_{i}, \alpha_{j}\right], \alpha_{0}, \ldots, \hat{\alpha}_{i}, \ldots, \hat{\alpha}_{j}, \ldots, \alpha_{n}\right) .
\end{aligned}
$$

We summarize the discussion up to this point in the following theorem.

Theorem 1. For any algebroid $A, C_{\mathrm{def}}^{n}(A)=\operatorname{Der}^{n-1}(A)$ and $\delta(D)=[m, D]$. In particular, $\left(C_{\mathrm{def}}^{*+1}(A), \delta\right)$ is a differential graded Lie algebra (note the shift in degree), and the shifted deformation cohomology $H_{\mathrm{def}}^{*+1}(A)$ is a graded Lie algebra.

\subsection{Alternative descriptions of the deformation complex}

There are several different descriptions of the space $\operatorname{Der}^{*}(E)$ of multiderivations on a vector bundle and of the deformation complex $C_{\text {def }}^{*}(A)$ of an algebroid. Here we give a description in terms of derivations (which reveals a connection between our deformation complex and recent unpublished work of D. Roytenberg), while in Subsection 4.9 we will give a more geometric description in terms of linear multivector fields. As before, we denote by $E$ a vector bundle over $M$, and we will change the notation to $A$ when dealing with Lie algebroids. 
By formulas similar to the classical ones for Lie derivatives of forms along vector fields, any derivation $D: \Gamma(E) \rightarrow \Gamma(E)$ induces an $\mathbb{R}$-linear derivation of degree zero on the algebra $C^{*}(E)=\Gamma\left(\bigwedge^{*} E^{\vee}\right)$ of sections of the exterior bundle (viewed as $C^{\infty}(M)$ valued, $C^{\infty}(M)$-multilinear maps on the powers of $\left.\Gamma(E)\right)$, by

$$
L_{D}(c)\left(s_{1}, \ldots, s_{q}\right)=L_{\sigma_{D}}\left(c\left(s_{1}, \ldots, s_{q}\right)\right)-\sum_{i=1}^{q} c\left(s_{1}, \ldots, D\left(s_{i}\right), \ldots, s_{q}\right)
$$

More generally, any $D \in \operatorname{Der}^{p}(E)$ induces a derivation of degree $p$ on $C^{*}(E)$ : if $c \in$ $C^{q}(E)$, then $L_{D}(c) \in C^{p+q}(E)$ is given by

$$
L_{D}(c)=(-1)^{p q} \sigma_{D} \circ c-c \circ D
$$

where $c \circ D$ is defined by the Gerstenhaber-type formula (2), and similarly,

$$
\sigma_{D} \circ c\left(s_{1}, \ldots, s_{p+q}\right)=\sum_{\sigma}(-1)^{|\sigma|} L_{\sigma_{D}\left(s_{\sigma(q+1)}, \ldots, s_{\sigma(p+q)}\right)}\left(c\left(s_{\sigma(1)}, \ldots, s_{\sigma(q)}\right)\right) .
$$

Here $L_{X}$ denotes the Lie derivative along any vector field $X$, and the sum is over all $(q, p)$-shuffles. Conversely, any $\mathbb{R}$-linear derivation of degree $p$ on $C^{*}(E)$ arises in this way, since it is uniquely determined by what it does on $C^{\infty}(M)$ and $\Gamma\left(E^{\vee}\right)$. For later reference we give the explicit formulas for $L_{D}$ applied to $f \in C^{0}(E)=C^{\infty}(M)$ and to $\xi \in C^{1}(E)=\Gamma\left(E^{\vee}\right)$, when $D \in \operatorname{Der}^{p-1}(E)$ :

$$
\begin{gathered}
L_{D}(f)\left(s_{1}, \ldots, s_{p-1}\right)=\sigma_{D}\left(s_{1}, \ldots, s_{p-1}\right)(f) \\
L_{D}(\xi)\left(s_{1}, \ldots, s_{p}\right)=\sum_{i=1}^{p}(-1)^{p-i} L_{\sigma_{D}\left(s_{1}, \ldots, \hat{s}_{i}, \ldots, s_{p}\right)}\left(\xi\left(s_{i}\right)\right)-\xi\left(D\left(s_{1}, \ldots, s_{p}\right)\right) .
\end{gathered}
$$

The conclusion is that $\operatorname{Der}^{*}(E)$ is isomorphic to the algebra of derivations of $C^{*}(E)$ (as graded Lie algebras!). With this, Lemma 2 translates into the following well-known observation [11].

Corollary 1. Given a vector bundle $E$, there is a one-to-one correspondence between Lie algebroid structures on $E$ and derivations $\delta$ of degree 1 on the algebra $C^{*}(E)=$ $\Gamma\left(\bigwedge E^{\vee}\right)$, satisfying $\delta^{2}=0$.

Note that if $E=A$ is a Lie algebroid, we obtain an action of $C_{\mathrm{def}}^{*}(A)$ on $C^{*}(A)$ (be aware of the degree shift!), and the differential $\delta_{A}$ of $C^{*}(A)$ coincides with $L_{m}$, where $m \in C_{\mathrm{def}}^{2}(A)$ is the Lie bracket of $A$. The discussion above and a careful computation of the boundaries shows that, conversely, one recovers the differential graded Lie algebra $\left(C_{\mathrm{def}}^{*}(A), \delta\right)$ as the algebra of derivations of the differential graded algebra $\left(C^{*}(A), \delta_{A}\right)$. We will come back to this point in Subsection 4.8 below. 


\section{Deformation cohomology and deformations}

In this section we examine our deformation cohomology of Lie algebroids in low degrees. In degree zero,

$$
H_{\mathrm{def}}^{0}(A)=Z(\Gamma(A)),
$$

the center of the infinite-dimensional Lie algebra of sections of $A$. In degrees 1 and 2 we will show that the usual interpretations for Lie algebras extend to the context of Lie algebroids. In particular, degree 2 cohomology classes will be seen to correspond to deformations, thus justifying the name "deformation cohomology".

\section{1. $H_{\mathrm{def}}^{1}$ and derivations}

Recall that a derivation of a Lie algebroid $A$ is a linear map $D: \Gamma(A) \rightarrow \Gamma(A)$ which is both a vector bundle derivation (see Subsection 2.1) and a derivation with respect to the Lie bracket:

$$
D([\alpha, \beta])=[D(\alpha), \beta]+[\alpha, D(\beta)] .
$$

These derivations form a Lie algebra $\operatorname{Der}(A)$ under the commutator bracket. This Lie algebra was studied in [15, 16]. The inner derivations, i.e. those of the form $[\alpha,-]$, form an ideal in $\operatorname{Der}(A)$, and the quotient, denoted $\operatorname{OutDer}(A)$, is the Lie algebra of outer derivations of $A$. It is immediate from the definitions that

$$
H_{\mathrm{def}}^{1}(A)=\operatorname{OutDer}(A)
$$

This space can also be interpreted as the Lie algebra of the (infinite-dimensional) group of outer automorphisms of $A$. For later use, we make this statement more precise. The passage from the infinitesimal side (derivations) to the global side (automorphisms) is via flows of derivations (see the Appendix in [4]). Given $D \in \operatorname{Der}(E)$, the flow $\Phi_{D}^{t}$ of $D$ is a 1-parameter group of bundle isomorphisms of $E$, covering the flow $\phi_{\sigma_{D}}^{t}$ of the symbol $\sigma_{D}$ of $D$ :

$$
\Phi_{D}^{t}(x): E_{x} \rightarrow E_{\phi_{\sigma_{D}}^{t}(x)}
$$

It is characterized uniquely by the property

$$
\left.\frac{d}{d t}\right|_{t=0}\left(\Phi_{D}^{t}\right)^{*} \beta=D(\beta), \quad \forall \beta \in \Gamma(E) .
$$

In this relation, $\left(\Phi_{D}^{t}\right)^{*}(\beta)(x)=\Phi_{D}^{t}\left(\beta\left(\phi_{\sigma_{D}}^{-t}(x)\right)\right)$. In general, $\Phi_{D}^{t}(x)$ will be defined whenever $\phi_{\sigma_{D}}^{t}(x)$ is, and one has to deal with local bundle maps, defined only over some open sets. Since this is standard, and not relevant for the present discussion, we will assume that the vector fields $\sigma_{D}$ are complete.

Lemma 4. Let $A$ be a Lie algebroid. A derivation $D \in \operatorname{Der}(A)$ is a Lie algebroid derivation if and only if the maps $\Phi_{D}^{t}$ are Lie algebroid automorphisms. 
The proof is standard. In the case of an inner derivation $D=\operatorname{ad}_{\alpha}$, one denotes $\Phi_{D}^{t}$ by $\Phi_{\alpha}^{t}$, and calls it the (infinitesimal) flow of $\alpha$. These flows play an essential role in [4], and they can be thought of as the inner automorphisms of $A$. Therefore, we can think of the Lie algebra $H_{\text {def }}^{1}(A)$ as the Lie algebra of the (infinite-dimensional) group of outer automorphisms of $A$.

\section{2. $H_{\mathrm{def}}^{2}$ and deformations}

We now explain the relevance of the deformation complex to deformations of Lie algebroids.

Definition 1. Let $A$ be a fixed vector bundle, and $I \subset \mathbb{R}$ an interval.

(i) A family of Lie algebroids over I is a collection $\left(A_{t}\right)_{t \in I}$ of Lie algebroids $A_{t}=$ $\left(A,[\cdot, \cdot]_{t}, \rho_{t}\right)$ varying smoothly with respect to $t$.

(ii) We say that two families $\left(A_{t}\right)_{t \in I}$ and $\left(A_{t}^{\prime}\right)_{t \in I}$ of Lie algebroids are equivalent if there exists a family of Lie algebroid isomorphisms $h_{t}: A_{t} \rightarrow A_{t}^{\prime}$, depending smoothly on $t$. Such a family $h$ is called an equivalence.

(iii) A deformation of a Lie algebroid $(A,[\cdot, \cdot], \rho)$ is a family $\left(A_{t}\right)_{t \in I}$ of Lie algebroids over an interval containing the origin with $A_{0}=A$.

(iv) Two deformations $\left(A_{t}\right)_{t \in I}$ and $\left(A_{t}^{\prime}\right)_{t \in I}$ of a Lie algebroid $A$ are said to be equivalent if there exists an equivalence $h_{t}: A_{t} \rightarrow A_{t}^{\prime}$ with $h_{0}=\mathrm{Id}$.

We will say that a family (or deformation) is trivial if it is equivalent to the constant family (deformation). We have the following interpretation for the elements of the second cohomology group $H_{\text {def }}^{2}(A)$ in terms of deformations.

Proposition 2. Let $A_{t}=\left(A,[\cdot, \cdot]_{t}, \rho_{t}\right)$ be a deformation of the Lie algebroid A. Then

$$
c_{0}(\alpha, \beta)=\left.\frac{d}{d t}[\alpha, \beta]_{t}\right|_{t=0}
$$

defines a cocycle $c_{0} \in C_{\mathrm{def}}^{2}(A)$. The corresponding cohomology class in $H_{\mathrm{def}}^{2}(A)$ only depends on the equivalence class of the deformation.

Proof. Let us denote, as before, by $m_{t} \in \operatorname{Der}^{1}(A)$ the Lie bracket $[\cdot, \cdot]_{t}$. Since $\operatorname{Der}^{1}(A)=$ $\Gamma\left(\mathcal{D}^{1}(A)\right), c_{0}$ is a multiderivation (its symbol is $\sigma=\left.\frac{d}{d t} \rho_{t}\right|_{t=0}$ ). Taking derivatives at $t=0$ in the equation $\left[m_{t}, m_{t}\right]=0$ we obtain $\left[c_{0}, m\right]=0$, i.e., $\delta\left(c_{0}\right)=0$ so that $c_{0}$ is a cocycle.

Assume now that $A_{t}^{\prime}$ is another deformation, and denote the associated class by $c_{0}^{\prime}$. Assume also that $h_{t}$ defines an equivalence between $A_{t}$ and $A_{t}^{\prime}$. We use the same notation $h_{t}$ for the map induced at the level of sections, and we consider the derivation $D$ defined by

$$
D=\left.\frac{d}{d t}\right|_{t=0} h_{t}: \Gamma(A) \rightarrow \Gamma(A) .
$$


Since $h_{t}$ is a Lie algebroid map we have

$$
h_{t}\left([\alpha, \beta]_{t}\right)=\left[h_{t}(\alpha), h_{t}(\beta)\right]_{t}^{\prime} .
$$

Taking derivatives of both sides and setting $t=0$, we obtain

$$
D([\alpha, \beta])+c_{0}(\alpha, \beta)=c_{0}^{\prime}(\alpha, \beta)+[D(\alpha), \beta]+[\alpha, D(\beta)],
$$

which means precisely that $c_{0}-c_{0}^{\prime}=\delta(D)$.

Remark 1. Note that, when talking about deformations, one can also allow the vector bundle $A$ itself to vary smoothly with respect to $t$ (in the sense that the $A_{t}$ 's together fit into a smooth vector bundle over $M \times \mathbb{R}$ ). Indeed, to any such family $A_{t}$ of algebroids, one can associate (an equivalence class of) a family of algebroids with constant vector bundle $A=A_{0}$, by choosing vector bundle isomorphisms $\phi_{t}: A_{t} \rightarrow A$ (which is possible because the real line is contractible) and transporting the bracket of $A_{t}$ to a bracket $[\cdot, \cdot]_{t}$ on $A$. Of course, different choices of $\phi_{t}$ produce equivalent deformations in the sense above. In particular, the cohomology class of the deformation is defined unambiguously for any deformation of $A$ with possibly varying vector bundle.

If $\left(A_{t}\right)_{t \in I}$ is a trivial deformation of a Lie algebroid $A$ then obviously we must have $\left[c_{0}\right]=0$. The converse is not true, but our next result gives a partial converse

Theorem 2. Let $\left(A_{t}\right)_{t \in I}=\left(A,[\cdot, \cdot]_{t}, \rho_{t}\right)$ be a family of Lie algebroids. Then

$$
c_{t}(\alpha, \beta)=\frac{d}{d t}[\alpha, \beta]_{t}
$$

defines a cocycle $c_{t} \in C_{\mathrm{def}}^{2}\left(A_{t}\right)$. If $M$ is compact, then the following are equivalent:

(i) The family $\left(A_{t}\right)_{t \in I}$ is trivial.

(ii) The classes $\left[c_{t}\right] \in H_{\mathrm{def}}^{2}\left(A_{t}\right)$ vanish smoothly with respect to $t$, i.e., $c_{t}=\delta\left(D_{t}\right)$ for $a$ smooth family $D_{t}$ of 1-cochains.

Proof. The fact that, for each $t, c_{t}$ is a cocycle and its cohomology class depends only on the equivalence class of $\left(A_{t}\right)_{t \in I}$ follows from Proposition 2 , since we can view $A_{s}$ as a deformation of $A_{t}$.

Let $\left(A_{t}\right)_{t \in I}$ be a trivial family, so that there exist Lie algebroid isomorphisms $h_{t}$ : $A_{t} \rightarrow A$ to a fixed Lie algebroid $A$. We define $D_{t}: \Gamma\left(A_{t}\right) \rightarrow \Gamma\left(A_{t}\right)$ by letting

$$
h_{t}\left(D_{t}(\alpha)\right)=\frac{d}{d t} h_{t}(\alpha) .
$$

Differentiating both sides of

$$
h_{t}\left([\alpha, \beta]_{t}\right)=\left[h_{t}(\alpha), h_{t}(\beta)\right],
$$

we obtain

$$
\begin{aligned}
h_{t}\left(D_{t}\left([\alpha, \beta]_{t}\right)+c_{t}(\alpha, \beta)\right) & =\left[h_{t}\left(D_{t}(\alpha)\right), h_{t}(\beta)\right]+\left[h_{t}(\alpha), h_{t}\left(D_{t}(\beta)\right)\right] \\
& =h_{t}\left(\left[D_{t}(\alpha), \beta\right]_{t}+\left[\alpha, D_{t}(\beta)\right]_{t}\right) .
\end{aligned}
$$


This shows that

$$
c_{t}(\alpha, \beta)=\left[D_{t}(\alpha), \beta\right]_{t}+\left[\alpha, D_{t}(\beta)\right]_{t}-D_{t}\left([\alpha, \beta]_{t}\right),
$$

which means precisely that $c_{t}=\delta\left(D_{t}\right)$, so the classes $\left[c_{t}\right] \in H_{\text {def }}^{2}\left(A_{t}\right)$ vanish smoothly with respect to $t$.

Conversely, suppose that $\left(A_{t}\right)_{t \in I}$ is a family of Lie algebroids such that $c_{t}=\delta\left(D_{t}\right)$ for a smooth family $D_{t}$ of 1-cochains. Each $D_{t}$ is a derivation, and since $M$ is compact, the flow $\Phi_{D_{t}}^{s}$ of $D_{t}$ is defined for all $s$, for each fixed $t$. Denote by $h_{t}$ the flow at time $t$. From the defining relation 10 ) for the flow of a derivation, we have

$$
h_{t}\left(D_{t}(\alpha)\right)=\frac{d}{d t} h_{t}(\alpha)
$$

We claim that

$$
h_{t}\left([\alpha, \beta]_{t}\right)=\left[h_{t}(\alpha), h_{t}(\beta)\right]_{0} .
$$

This shows that $h_{t}: A_{t} \rightarrow A_{0}$ gives an equivalence to a constant family, so the family $\left(A_{t}\right)_{t \in I}$ will be trivial.

To prove the claim, we just observe that (11) holds at $t=0$, and that the derivative of both sides of (11) are equal. In fact, as we saw above, the derivative is precisely the condition $c_{t}=\delta\left(D_{t}\right)$. Therefore equality holds for all $t$.

\section{Relations to known cohomologies and particular cases}

In this section we look at some particular classes of algebroids, and we relate $H_{\text {def }}^{*}(A)$ to known cohomology theories. We begin by simply mentioning the following two extreme cases.

\subsection{Lie algebras}

Since the Lie algebra case was partially used as inspiration for our constructions, it is clear that the deformation complex $C_{\text {def }}^{*}(\mathfrak{g})$ of a Lie algebra $\mathfrak{g}$ is the usual Chevalley-Eilenberg complex $C^{*}(\mathfrak{g}, \mathfrak{g})$ with coefficients in the adjoint representation, and one recovers the classical relation between Lie algebra deformations, the graded Lie algebra $C^{*}(\mathfrak{g}, \mathfrak{g})$, and the cohomology groups $H^{*}(\mathfrak{g}, \mathfrak{g})[18,19]$.

\subsection{Tangent bundles}

Let us now look at the case where $A=T M$ is the tangent bundle of a manifold $M$. The first remark is that closed cocycles correspond to vector-valued forms on $M$ :

$$
Z^{k} C_{\text {def }}^{*}(T M) \cong \Gamma\left(\bigwedge^{k-1} T^{\vee} M \otimes T M\right),
$$


where $T^{\vee} M$ denotes the cotangent bundle. This follows from 4 which shows that any cocycle $D$ in the deformation complex is determined by its symbol $\sigma_{D}$ by $D=$ $(-1)^{k} \delta\left(\sigma_{D}\right)$. Actually, the same formula shows that the map which associates to $D$ its symbol, viewed as a map of degree -1 on $C_{\text {def }}^{*}(T M)$, defines a homotopy in the deformation complex (up to a sign). Hence we recover the following result of [6]:

Corollary 2. Any closed multiderivation on $T M$ is exact (i.e. $H_{\mathrm{def}}^{*}(T M)=0$ ).

A different (and more general) argument will be presented in Subsection 4.7 below.

\subsection{Cotangent bundles}

We now consider the graded Lie algebra of multiderivations on the cotangent bundle $T^{\vee} M$ of a manifold $M$, and we relate it to the known graded Lie algebra of multivector fields on $T M,\left(\mathcal{X}^{*}(M),[\cdot, \cdot]\right)$. Recall that $\mathcal{X}^{p}(M)=\bigwedge^{p}(T M)$, where the Lie algebra degree of a $p$-vector field is $p-1$, so that the graded antisymmetry reads

$$
[X, Y]=-(-1)^{(p-1)(q-1)}[Y, X]
$$

for $X \in \mathcal{X}^{p}(M), Y \in \mathcal{X}^{q}(M)$. Recall also that $[\cdot, \cdot]$ is the Nijenhuis-Schouten bracket; explicitly, for $X_{i}, Y_{j} \in \mathcal{X}(M)$ and $f \in C^{\infty}(M)$,

$$
\begin{aligned}
{\left[X_{1} \wedge \cdots \wedge X_{p}, Y_{1} \wedge \cdots \wedge Y_{q}\right] } & \\
= & \sum_{i, j}(-1)^{i+j}\left[X_{i}, Y_{j}\right] \wedge X_{1} \wedge \cdots \wedge \hat{X}_{i} \wedge \cdots \wedge X_{p} \wedge Y_{1} \wedge \cdots \wedge \hat{Y}_{j} \wedge \cdots \wedge Y_{q} \\
& {\left[X_{1} \wedge \cdots \wedge X_{p}, f\right]=\sum_{i=1}^{p}(-1)^{p-i} L_{X_{i}}(f) X_{1} \wedge \cdots \wedge \hat{X}_{i} \wedge \cdots \wedge X_{p} . }
\end{aligned}
$$

To state the next proposition, we also need the following notation: for $X \in \mathcal{X}^{n}(M)$, we view $X$ as an antisymmetric map depending on $n 1$-forms $\omega_{1}, \ldots, \omega_{n}$. Fixing the first $n-1$ of them defines a linear map $X\left(\omega_{1}, \ldots, \omega_{n-1},-\right)$ on $T^{\vee} M$, hence a vector field. We denote this vector field by $X^{\sharp}\left(\omega_{1}, \ldots, \omega_{n-1}\right)$, so that $X^{\sharp}$ becomes a linear antisymmetric map

$$
X^{\sharp}: \underbrace{T^{\vee} M \otimes \cdots \otimes T^{\vee} M}_{n-1 \text { times }} \rightarrow T M .
$$

Proposition 3. For any $X \in \mathcal{X}^{n}(M)$, there exists a unique $D_{X} \in \operatorname{Der}^{n-1}\left(T^{\vee} M\right)$ with symbol $X^{\sharp}$ and satisfying

$$
D_{X}\left(d f_{1}, \ldots, d f_{n}\right)=d\left(X\left(d f_{1}, \ldots, d f_{n}\right)\right)
$$

for all $f_{i} \in C^{\infty}(M)$. Explicitly, for all $\omega_{i} \in \Omega^{1}(M), 1 \leq i \leq n$,

$$
D_{X}\left(\omega_{1}, \ldots, \omega_{n}\right)=\sum_{i=1}^{n}(-1)^{n-i} L_{X^{\sharp}\left(\omega_{1}, \ldots, \hat{\omega}_{i}, \ldots, \omega_{n}\right)}\left(\omega_{i}\right)-(n-1) d\left(X\left(\omega_{1}, \ldots, \omega_{n}\right)\right) .
$$


Moreover, the map $\mathcal{X}(M) \rightarrow \operatorname{Der}\left(T^{\vee} M\right), X \mapsto D_{X}$, is a map of graded Lie algebras which is injective in strictly positive degrees. (For $n=0$, the convention is that $D_{X}=d(X)$.)

Proof. The uniqueness part is clear. That the explicit formula for $D_{X}$ defines a multiderivation with the desired properties follows by direct computation. The injectivity is immediate because the symbol of $D_{X}$ is $X^{\sharp}$. To prove that $X \mapsto D_{X}$ is compatible with the Lie bracket, we recall first that $\left(\mathcal{X}^{*}(M), \wedge,[\cdot, \cdot]\right)$ is a Gerstenhaber algebra, where $\wedge$ is the exterior product. Apart from the fact that $\left(\mathcal{X}^{*}(M),[\cdot, \cdot]\right)$ is a graded Lie algebra (with the Lie algebra degree of $X \in \mathcal{X}^{p}(M)$ equal to $p-1$ ), this also means that $\left(\mathcal{X}^{*}(M), \wedge\right)$ is a graded algebra (without degree shift!), while the two structures are related by the Leibniz rule:

$$
[X, Y \wedge Z]=[X, Y] \wedge Z+(-1)^{(p-1) q} Y \wedge[X, Z]
$$

for all $X \in \mathcal{X}^{p}(M), Y \in \mathcal{X}^{q}(M), Z \in \mathcal{X}^{r}(M)$. More formally, this equation means that the map $X \mapsto[X,-]$ is a graded map from $\left(\mathcal{X}^{*}(M),[\cdot, \cdot]\right)$ into the graded Lie algebra $\operatorname{Der}\left(\mathcal{X}^{*}(M), \wedge\right)$ of derivations on the graded algebra $\left(\mathcal{X}^{*}(M), \wedge\right)$.

On the other hand, by 2.5 applied to $T^{\vee} M$, we have an isomorphism $D \mapsto L_{D}$ from the graded Lie algebra $\operatorname{Der}\left(T^{\vee} M\right)$ of multiderivations on the cotangent bundle into the graded Lie algebra of derivations on $\left(\mathcal{X}^{*}(M), \wedge\right)$. We claim that $L_{D_{X}}=[X,-]$. Since this is an equality of derivations on $\left(\mathcal{X}^{*}(M), \wedge\right)$, it suffices to show that $L_{D_{X}}$ and $[X,-]$ are equal on functions and on vector fields. This follows again by direct computation, using the formulas for the Nijenhuis-Schouten bracket given above, and the formulas (8), (9) describing $L_{D}$ in low degrees for $D=D_{X}$.

Note that the injectivity of the map $X \mapsto D_{X}$ can also be seen as a special case of Proposition 5.1 of [20].

\subsection{Poisson manifolds I}

Here we describe the relation of our deformation cohomology with Poisson cohomology. Recall that a Poisson manifold is a pair $(P, \pi)$ where $\pi \in \Gamma\left(\bigwedge^{2}(T P)\right)$ is a bivector with the property that the "Poisson bracket" $\{f, g\}=\pi(d f, d g)$ on $C^{\infty}(P)$ satisfies the Jacobi identity, or equivalently, the Nijenhuis-Schouten bracket $[\pi, \pi]$ vanishes. It is well-known that a Poisson structure on $P$ induces an algebroid structure on $T^{\vee} P$ with anchor $\pi^{\sharp}$ (defined by $\beta\left(\pi^{\sharp}(\alpha)\right)=\pi(\alpha, \beta)$ for all 1 -forms $\alpha$ and $\beta$ ). The bracket is usually introduced either by the explicit formula

$$
[\alpha, \beta]=L_{\pi^{\sharp}(\alpha)}(\beta)-L_{\pi^{\sharp}(\beta)}(\alpha)-d(\pi(\alpha, \beta)),
$$

or by saying that it is the unique Lie algebroid structure on $T^{\vee} P$ with anchor $\pi^{\sharp}$ and the property that $[d f, d g]=d\{f, g\}$.

It is interesting to relate this to the previous proposition. Since $[\pi, \pi]=0$, the induced derivation $D_{\pi} \in \operatorname{Der}^{1}\left(T^{\vee} P\right)$ satisfies the same formula, hence it defines an algebroid structure on $T^{\vee} P$ by Lemma 2 . This coincides with the known algebroid structure, 
and the two ways of describing the structure correspond to the descriptions of $D_{\pi}$ in Proposition 3

Next, given the Poisson manifold $P$, the de Rham cohomology of the induced Lie algebroid $T^{\vee} P$ (with coefficients in the trivial line bundle) is known as the Poisson cohomology of $P$, denoted $H_{\pi}^{*}(P)$. The defining complex is the complex of multivector fields

$$
C_{\pi}^{*}(P)=\Gamma\left(\bigwedge^{*} T P\right),
$$

with boundary $d(X)=[\pi, X]$, where $[\cdot, \cdot]$ is the Nijenhuis-Schouten bracket (see e.g. [22] and the references therein). In particular, $C_{\pi}^{*}(P)$ is a dgla.

Finally, if $\pi_{t}$ is a family of Poisson structures on $P$ with $\pi_{0}=\pi$, then taking derivatives with respect to $t$ in $\left[\pi_{t}, \pi_{t}\right]=0$ at $t=0$, we see that

$$
\left[\left.\frac{d}{d t}\right|_{t=0} \pi_{t}\right] \in H_{\pi}^{2}(P)
$$

is a well-defined cohomology class. This is known as the cohomology class associated to the deformation $\pi_{t}$. Using the last part of Proposition 3 and the remark above that $D_{\pi}$ is the Lie bracket of $T^{\vee} P$, we deduce:

Corollary 3. For any Poisson manifold $(P, \pi)$, the map $X \mapsto D_{X}$ is a dg Lie morphism from the Poisson complex $C_{\pi}^{*}(P)$ into the deformation complex $C_{\mathrm{def}}^{*}\left(T^{\vee} P\right)$, which is injective in positive degrees. In particular, there is an induced map of graded Lie algebras

$$
i: H_{\pi}^{*}(P) \rightarrow H_{\mathrm{def}}^{*}\left(T^{\vee} P\right) .
$$

Moreover, if $\left\{\pi_{t}\right\}$ is a deformation of $\pi$, then $[\cdot, \cdot]_{\pi_{t}}$ defines a deformation of the Lie algebroid $\left(T^{\vee} P,[\cdot, \cdot]_{\pi}\right)$, and the associated cohomology classes (i.e. $(12)$, and the one of Proposition 2 respectively) are related by the map $i$.

\subsection{Foliations}

We now look at the particular case of regular foliations on a manifold $M$, i.e. subbundles $\mathcal{F} \subset T M$ (of vectors tangent to the leaves) which are involutive (i.e. $[\Gamma(\mathcal{F}), \Gamma(\mathcal{F})] \subset$ $\Gamma(\mathcal{F})$ ). Regular foliations are the same thing as Lie algebroids with injective anchor map. Similar to the adjoint representation of a Lie algebra, any foliation $\mathcal{F}$ has a canonical representation on the normal bundle $v=T M / \mathcal{F}$, called the Bott representation, defined by $\nabla_{X}(\bar{Y})=\overline{[X, Y]}$. The resulting cohomology $H^{*}(\mathcal{F} ; v)$ is known as the foliated (or leafwise) cohomology with coefficients in the normal bundle, and was investigated by Heitsch [10] in connection with deformations of foliations. Explicitly, given a family of foliations $\mathcal{F}_{t}$ with $\mathcal{F}_{0}=\mathcal{F}$, Heitsch defines

$$
c_{0}(v)=\pi_{0}^{\perp}\left(\left.\frac{d}{d t}\right|_{t=0} \pi_{t}(v)\right)
$$


for all $v \in \Gamma(\mathcal{F})$, where $\pi_{t}: T M \rightarrow \mathcal{F}_{t}$ and $\pi_{t}^{\perp}: T M \rightarrow v_{t}$ are the orthogonal projections with respect to a Riemannian metric. Then $c_{0}$ defines a cohomology class

$$
\left[c_{0}\right] \in H^{1}(\mathcal{F} ; v),
$$

independent of the metric, which we will call the Heitsch characteristic class of the deformation.

On the other hand, each such deformation defines a deformation of $\mathcal{F}$ viewed as an algebroid, hence a class in $H_{\text {def }}^{2}(\mathcal{F})$. We have:

Proposition 4. For any foliation $\mathcal{F}$,

$$
H_{\text {def }}^{*}(\mathcal{F}) \cong H^{*-1}(\mathcal{F} ; v) .
$$

Moreover, for a deformation $\mathcal{F}_{t}$ of $\mathcal{F}$, the induced cohomology class in $H_{\text {def }}^{2}(\mathcal{F})$ corresponds to the Heitsch characteristic class of the deformation.

Note that, in degree zero,

$$
l(M, \mathcal{F}):=H^{0}(\mathcal{F} ; v)
$$

is well-known in foliation theory as the Lie algebra of transversely projectable vector fields. It consists of the sections $\bar{X}$ of the normal bundle with the property that $[X, \Gamma(\mathcal{F})]$ $\subset \Gamma(\mathcal{F})$. The previous proposition together with Theorem 1 implies that the Lie algebra structure on $l(M, \mathcal{F})$ is just the degree zero part of a graded Lie algebra structure on $H^{*}(\mathcal{F} ; v)$.

Proof of Proposition 4. The first part of the proposition will be immediate from Theorem 3 below. Here we sketch a different argument, similar to the one in the case of tangent bundles. Note first that equation $\left(4 \mathrm{r}\right.$ implies that if $D \in C_{\text {def }}^{k}(\mathcal{F})$ is a cocycle, then $D$ is uniquely determined by its symbol $\sigma_{D} \in C^{k-1}(\mathcal{F} ; T M)$. On the other hand, the symbol of $D$ projects to an element $\bar{\sigma}_{D} \in C^{k-1}(\mathcal{F} ; v)$ which will be a cocycle. One then checks directly that the induced cohomology class only depends on the cohomology class of $D$, and this defines the desired isomorphism. (Alternatively, one can easily chase the sequences appearing in the proof of Theorem 3 .)

Let us now go to the second part. We are in the case described in Remark 1 where the vector bundle is varying also, hence we first need to trivialize $\mathcal{F}_{t}$ as a family of vector bundles, and then consider the induced brackets $[\cdot, \cdot]_{t}$ on $\mathcal{F}$. The trivialization we will use is the one induced by the parallel transport (with respect to $t$ ) of the canonical partial connection $\nabla_{d / d t}=\pi_{t} \circ \frac{d}{d t}$ on $\left(\mathcal{F}_{t}\right)_{t}$ viewed as a bundle over $M \times \mathbb{R}$. Since the induced trivialization $T_{t}: \mathcal{F} \rightarrow \mathcal{F}_{t}$ sends $v$ into its parallel transport $T_{t}(v)$ at time $t$, it is the solution of the equation

$$
\pi_{t}\left(\frac{d}{d t} T_{t}(v)\right)=0, \quad T_{0}(v)=v .
$$


The deformation cocycle induced by the deformation is $c=\left.\frac{d}{d t}\right|_{t=0}[\cdot, \cdot]_{t}$. From the first part, we know that the cocycle $\sigma \in C^{1}(\mathcal{F} ; v)$ corresponding to $c$ is the symbol of $c$ (i.e. the anchor $T_{t}$ of $[\cdot, \cdot]_{t}$ ) projected down to $v$ :

$$
\sigma(v)=\pi_{0}^{\perp}\left(\left.\frac{d}{d t}\right|_{t=0} T_{t}(v)\right) \in \Gamma(v) .
$$

We claim that this coincides with $c_{0}$ of (13) defining the Heitsch class. Since both $c_{0}(v)$ and $\sigma(v)$ are tangent to $\mathcal{F}$, we only have to show that the orthogonal projection $\pi_{0}^{\perp}$ onto $\mathcal{F}$ kills their difference. This follows immediately by taking derivatives in $\pi_{t}^{\perp}\left(T_{t}(v)-\right.$ $\left.\pi_{t}(v)\right)=0$ at $t=0$.

\subsection{Lie algebra actions on manifolds}

Given an action of a Lie algebra $\mathfrak{g}$ on a manifold $M$, i.e. a Lie algebra map $\rho_{M}: \mathfrak{g} \rightarrow$ $\mathcal{X}(M)$, one has an induced Lie algebroid structure on $\mathfrak{g} \times M$ (the trivial vector bundle over $M$ with fiber $\mathfrak{g})$ : the anchor is the map $\rho$ defining the action, while the bracket is determined by its values on constant sections (the bracket between the constant sections $v, w \in \mathfrak{g} \subset \Gamma(\mathfrak{g} \times M)$ is the constant section $[v, w])$, and the Leibniz rule. The resulting algebroid is denoted by $\mathfrak{g} \ltimes M$ and is called the action algebroid associated to the infinitesimal action of $\mathfrak{g}$ on $M$. Such algebroids (and the study of their deformations) are important in the linearization problem of Poisson manifolds, although the case where $\mathfrak{g}$ is 1-dimensional is already interesting (the action on $M$ will be described by a vector field $X$ on $M$, and the integration of the associated action algebroid is nothing but the integration of the vector field $X$ ).

Such an action algebroid $A=\mathfrak{g} \ltimes M$ has two canonical representations. First of all, there is a natural action on the trivial vector bundle over $M$ with fiber $\mathfrak{g}, \mathfrak{g}_{M}=\mathfrak{g} \times M$, given by the unique $A$-connection $\nabla$ with the property that $\nabla_{v}(w)=[v, w]$, the bracket of $\mathfrak{g}$, for all $v \in \mathfrak{g}$ (viewed as constant sections of $A$ ) and $w \in \mathfrak{g}$ (viewed as constant sections of the representation). Note that the de Rham cohomology of $A$ with coefficients in this representation is

$$
H^{*}\left(A ; \mathfrak{g}_{M}\right)=H^{*}\left(\mathfrak{g} ; \Gamma\left(\mathfrak{g}_{M}\right)\right),
$$

the cohomology of the Lie algebra $\mathfrak{g}$ with coefficients in $\Gamma\left(\mathfrak{g}_{M}\right)=C^{\infty}(M ; \mathfrak{g})$.

Next, there is a similar action of $A$ on $T M$, where the connection is determined by $\nabla_{v}(X)=[\rho(v), X]$ for $v \in \mathfrak{g}$. The resulting cohomology is isomorphic to the cohomology of $\mathfrak{g}$ with coefficients in $\mathcal{X}(M)$ :

$$
H^{*}(A ; T M)=H^{*}(\mathfrak{g} ; \mathcal{X}(M)) .
$$

Proposition 5. For any action of a Lie algebra $\mathfrak{g}$ on a manifold $M$, the deformation cohomology of the associated action algebroid $A=\mathfrak{g} \ltimes M$ fits into a long exact sequence

$$
\cdots \rightarrow H^{n-1}(A ; T M) \rightarrow H_{\mathrm{def}}^{n}(A) \rightarrow H^{n}\left(A ; \mathfrak{g}_{M}\right) \stackrel{\delta}{\rightarrow} H^{n}(A ; T M) \rightarrow \cdots
$$


Proof. We have a short exact sequence

$$
0 \rightarrow C^{n-1}(A ; T M) \rightarrow C_{\mathrm{def}}^{n}(A) \stackrel{\pi}{\rightarrow} C^{n}\left(A ; \mathfrak{g}_{M}\right) \rightarrow 0,
$$

where $\pi$ associates to $D \in C_{\mathrm{def}}^{n}(A)$ the unique $\pi(D)=c \in C^{n}\left(A ; \mathfrak{g}_{M}\right)$ such that $D\left(v_{1}, \ldots, v_{n}\right)=c\left(v_{1}, \ldots, v_{n}\right)$ on constant sections.

\subsection{The regular case}

We now relate the deformation cohomology of $A$ to the de Rham cohomology of $A$ with coefficients, in the case where $A$ is regular, i.e. when $\rho$ has constant rank. In this case the image of $\rho$ defines a (regular) foliation $\mathcal{F}$ of $M$, and the isotropy bundle and the normal bundle,

$$
\mathfrak{g}(A)=\operatorname{Ker}(\rho), \quad v=T M / \mathcal{F},
$$

are both representations of $A$ : the first with $\nabla_{\alpha}(\beta)=[\alpha, \beta]$, and the second with the Bott connection $\nabla_{\alpha}(\bar{X})=\overline{[\rho(\alpha), X]}$. With these notations, we will show:

Theorem 3. For any regular Lie algebroid A, there is an associated long exact sequence

$$
\cdots \rightarrow H^{n}(A ; \mathfrak{g}(A)) \rightarrow H_{\mathrm{def}}^{n}(A) \rightarrow H^{n-1}(A ; \nu) \stackrel{\delta}{\rightarrow} H^{n+1}(A ; \mathfrak{g}(A)) \rightarrow \cdots,
$$

where, as above, $\mathfrak{g}$ is the isotropy Lie algebra of $A$, and $v$ is the normal bundle of the foliation induced by $A$.

Proof. To prove the theorem, we will introduce two auxiliary complexes $C_{1}^{*}$ and $C_{2}^{*}$ which fit into exact sequences of cochain complexes:

$$
\begin{array}{r}
0 \rightarrow C^{*}(A ; \mathfrak{g}(A)) \stackrel{j}{\rightarrow} C_{\mathrm{def}}^{*}(A) \stackrel{\rho}{\rightarrow} C_{1}^{*} \rightarrow 0, \\
0 \rightarrow C_{1}^{*} \stackrel{i}{\rightarrow} C_{2}^{*} \stackrel{\pi}{\rightarrow} C^{*}(A ; v) \rightarrow 0
\end{array}
$$

and such that $C_{2}^{*}$ has zero cohomology. Then the long exact sequence associated to 15 implies that $H^{n}\left(C_{1}^{*}\right) \cong H^{n-1}(A ; v)$, which when plugged into the long exact sequence associated to 14 will prove our theorem.

First of all, $C_{2}^{n}$ consists of the antisymmetric multilinear maps

$$
D: \underbrace{\Gamma(A) \otimes \cdots \otimes \Gamma(A)}_{n \text { times }} \rightarrow \Gamma(T M)
$$

together with $\sigma_{D} \in \Gamma\left(\bigwedge^{n-1} A^{\vee} \otimes T M\right)$ (the symbol of $\left.D\right)$, such that

$$
D\left(v_{1}, v_{2}, \ldots, f v_{n}\right)=f D\left(v_{1}, v_{2}, \ldots, v_{n}\right)+\sigma_{D}\left(v_{1}, \ldots, v_{n-1}\right)(f) \rho\left(v_{n}\right)
$$

for all functions $f$ and all sections $v_{i}$ (when $\rho \neq 0$ then, just as for multiderivations, $\sigma_{D}$ is uniquely determined by $D$ ). Note the similarity with $C_{\text {def }}^{*}(A)$, which we complete by defining the differential $\delta$ on $C_{2}^{*}$ by the same formula as in (1) and by equation (4). Next, 
$C_{1}^{*}$ is defined as the subcomplex of $C_{2}^{*}$ consisting of those $D$ which are $\Gamma(\mathcal{F})$-valued, and the maps in the exact sequences above are the obvious ones. The exactness of the sequences is evident, except maybe for the right hand side of (14) that we now explain. Let $D \in C_{1}^{*}$. Due to the exactness of the sequence in Lemma 1, we find $D^{\prime} \in C_{\mathrm{def}}^{n}(A)$ whose symbol $\sigma_{D^{\prime}}$ coincides with $\sigma_{D}$. Then $D-\rho\left(D^{\prime}\right)$ is multilinear, i.e. comes from a vector bundle map $\bigwedge^{n} A \rightarrow \mathcal{F}$. Since $\rho: A \rightarrow \mathcal{F}$ is surjective, we find a map $a: \bigwedge^{n} A \rightarrow A$ such that $D-\rho\left(D^{\prime}\right)=\rho(a)$, hence $D=\rho\left(D^{\prime}+a\right)$.

To prove that $C_{2}^{*}$ is acyclic, we remark that any $a \in \Gamma\left(\bigwedge^{n-1} A^{\vee} \otimes T M\right)$ can be viewed as an element in $C_{2}^{n-1}$ with zero symbol, and $\delta(a) \in C_{2}^{n}$ has as symbol $\sigma_{\delta(a)}=(-1)^{n} a$. Assume now that $D \in C_{2}^{n}$ is a cocycle. Then $D^{\prime}=D+(-1)^{n-1} \delta\left(\sigma_{D}\right) \in C_{2}^{n}$ will have $\sigma_{D^{\prime}}=0$, by the previous formula applied to $a=\sigma_{D}$. Hence we can apply the same formula to $D^{\prime}$ to deduce $D^{\prime}=0$, since $\delta\left(D^{\prime}\right)=0$. In conclusion, $D=(-1)^{n} \delta\left(\sigma_{D}\right)$, and $D$ is exact.

In the case of transitive Lie algebroids (i.e. with surjective anchor), we deduce

Corollary 4. For any transitive algebroid A,

$$
H_{\mathrm{def}}^{*}(A) \cong H^{*}(A ; \mathfrak{g}(A))
$$

\subsection{The action on de Rham cohomology}

As we have already seen in Subsection 2.5, the deformation complex $C_{\text {def }}^{*}(A)$ acts on the complex $C^{*}(A)$ computing the de Rham cohomology of the Lie algebroid $A$. More generally, it acts on all complexes $C^{*}(A ; E)$ with $E$ a representation of $A$ : one keeps the same formulas as in 2.5, except that one replaces the Lie derivatives $L$. (of smooth functions) in (7) by the covariant derivatives $\nabla$ (of sections of $E$ ). Again, one obtains an action of $C_{\mathrm{def}}^{*}(A)$ on $C^{*}(A ; E)$. Passing to cohomology, we obtain:

Proposition 6. For any representation $E$ of the Lie algebroid A, there is an induced action

$$
H_{\mathrm{def}}^{p+1}(A) \otimes H^{q}(A ; E) \rightarrow H^{p+q}(A ; E), \quad(D, c) \mapsto L_{D}(c),
$$

which makes $H^{*}(A ; E)$ into a graded module over the graded Lie algebra $H_{\mathrm{def}}^{*}(A)$.

When $D \in H_{\mathrm{def}}^{1}(A), L_{D}$ is given by the standard formula $(6)$. There is another interesting particular case of this action, which one obtains by looking at deformations of $A$. In general, any deformation $\left(A_{t}\right)$ of $A$ induces a "variation map" in cohomology

$$
\partial: H^{*}(A) \rightarrow H^{*+1}(A)
$$

as follows: given a cocycle $c \in C^{k}(A)$, one deforms it to a family of cochains $c_{t} \in$ $C^{*}\left(A_{t}\right)$; one remarks that

$$
\left.\frac{d}{d t}\right|_{t=0} \delta_{A_{t}}\left(c_{t}\right) \in C^{k+1}(A)
$$


is a cocycle (take the derivative at $t=0$ of $\delta_{A_{t}}^{2}\left(c_{t}\right)=0$ ), and denote by $\partial([c])$ the resulting cohomology class. It is not difficult to see that $\partial$ is nothing but the action $L_{c_{0}}$ on cohomology, where $c_{0} \in H_{\mathrm{def}}^{2}(A)$ is the cohomology class induced by the deformation $A_{t}$ of $A$ (see Proposition 2). In particular, this shows that the description above for $\partial$ does not depend on the choices one makes, and it only depends on the equivalence class of the deformation.

\subsection{Poisson manifolds II}

There is yet another relation between Lie algebroids and Poisson manifolds: Lie algebroid structures on the vector bundle $A$ are in one-to-one correspondence with Poisson structures on its dual $A^{\vee}$ which are "linear on the fibers" [2]. The deformation complex gives a conceptual interpretation (and a new proof) of this result, which, as we shall now explain, implies that the deformation cohomology of $A$ coincides with the "lin-Poisson cohomology" of $A^{\vee}$.

To this end, we will look at vector bundles as being manifolds with a certain partial linear structure and use the ideas of Mackenzie on double structures [14, 15]. Accordingly, vector bundles will be referred to as lin-manifolds, and the category of vector bundles (with varying base!) will be called the lin-category. Hence a lin-manifold $E$ has an underlying manifold $E_{0}$ over which it is a vector bundle. Many of the classical objects have a corresponding lin-version. For instance, given a lin-manifold $E$, we can talk about linvector bundles $\mathcal{E}$ over $E$ : the addition will be a map $\mathcal{E} \times_{E} \mathcal{E} \rightarrow \mathcal{E}$ in the lin-category. In Mackenzie's terminology, $\mathcal{E}$ will be a double vector bundle: over $E$ and over $\mathcal{E}_{0}$, which are both vector bundles over $M=E_{0}$. The space $\Gamma_{\text {lin }}(\mathcal{E})$ is defined as the space of sections $s: E \rightarrow \mathcal{E}$ which are morphisms in the lin-category (hence lie over a section $s_{0}: E_{0} \rightarrow \mathcal{E}_{0}$ of the vector bundle $\mathcal{E}_{0}$ ).

There are two important examples: the tangent bundle $T E$ of a lin-manifold $E$ is naturally a lin-vector bundle over $E$ with $(T E)_{0}=T\left(E_{0}\right)$, while the cotangent bundle $T^{\vee} E$ is a lin-vector bundle over $E$ with $\left(T^{\vee} E\right)_{0}=E^{\vee}$ (where the projection $T^{\vee} E \rightarrow E^{\vee}$ comes from the inclusion $\pi^{*} E \subset T E, \pi: E \rightarrow M$ being the projection). Sections of $T E$ and $T^{\vee} E$ in the lin-category will define the space $\mathcal{X}_{\operatorname{lin}}(E)$ of linear vector fields on $E$, and $\Omega_{\text {lin }}^{1}(E)$ of linear 1-forms on $E$ (see also [15]). For instance, an element in $\mathcal{X}_{\text {lin }}(E)$ consists of a vector field $X$ on $E$ and a vector field $X_{0}$ on $M$ such that $X: E \rightarrow T E$ is a vector bundle map over $X_{0}: M \rightarrow T M$. Of course, $\mathcal{X}_{\text {lin }}(E) \subset \mathcal{X}(E)$, and, locally, (with respect to local coordinates $x_{i}$ in $M$ and a basis $e_{i}$ in $E$ ), the linear vector fields are the vector fields on $E$

$$
\sum a_{i} \frac{\partial}{\partial x_{i}}+\sum b_{j} \frac{\partial}{\partial e_{j}}
$$

with the property that $a_{i}=a_{i}(x)$ depends only on $x \in M$, and $b_{j}=b_{j}(x, v)$ is linear in $v$. In the same way we can talk about the space $\Gamma_{\text {lin }}(\mathcal{E})$ of any lin-vector bundle $\mathcal{E}$ over $E$, so that

$$
\mathcal{X}_{\operatorname{lin}}(E)=\Gamma_{\operatorname{lin}}(T E), \quad \Omega_{\operatorname{lin}}^{1}(E)=\Gamma_{\operatorname{lin}}\left(T^{\vee} E\right) .
$$


Note also that $\Omega_{\text {lin }}^{1}(E) \subset \Omega^{1}(E)$ consists of those 1-forms $\omega$ on $E$ with the property that $\omega(X) \in C_{\text {lin }}^{\infty}(E)$ for all $X \in \mathcal{X}_{\text {lin }}(E)$. We define $\Omega_{\text {lin }}^{k}(E) \subset \Omega^{k}(E)$ by the similar property: when applied to linear vector fields, it must produce linear smooth functions. And, dually, pairing multivector fields with wedge products of 1 -forms, we define the spaces $\mathcal{X}_{\text {lin }}^{k}(E)$ of linear $k$-vector fields on $E$. In local coordinates, these are vector fields which are sums of vectors of type

$$
a(x) \frac{\partial}{\partial x_{i_{1}}} \ldots \frac{\partial}{\partial x_{i_{k}}}+\sum b(x, v) \frac{\partial}{\partial x_{i_{1}}} \ldots \frac{\partial}{\partial x_{i_{k-1}}} \frac{\partial}{\partial e_{j}},
$$

with $b(x, v)$ linear in $v$. Note that these spaces are closed under the Nijenhuis-Schouten bracket on multivector fields, denoted by $[\cdot, \cdot]$.

According to the general philosophy, a linear Poisson structure on a vector bundle $E$ over $M$ is a Poisson structure whose Poisson tensor $\pi$ is linear: $\pi \in \mathcal{X}_{\text {lin }}^{2}(E)$. Also, the space of linear-multivector fields on $E, \mathcal{X}_{\text {lin }}(E)$, defines a subcomplex of the Poisson complex of $E$, and we define the linear Poisson cohomology of $E$, denoted $H_{\pi \text {,lin }}^{*}(E)$, as the cohomology of the resulting complex.

Next, any $X \in \mathcal{X}\left(E^{\vee}\right)$ induces a multiderivation $D_{X}$ with

$$
D_{X}\left(s_{1}, \ldots, s_{k}\right)=\left(d s_{1} \wedge \cdots \wedge d s_{k}\right)(X),
$$

where, for $s \in \Gamma(E)$, one views $s$ as a linear smooth function on $E^{\vee}$, and $d s \in \Omega_{\text {lin }}^{1}\left(E^{\vee}\right)$ is its differential. The symbol $\sigma_{X}$ of $D_{X}$ is determined by

$$
d \phi\left(\sigma_{X}\left(s_{1}, \ldots, s_{k-1}\right)\right)=\left(d s_{1} \wedge \cdots \wedge d s_{k-1} \wedge d \phi\right)(X)
$$

where, as before, for $\phi \in C^{\infty}(M)$, we denote by the same letter the function induced on $E^{\vee}$ (constant on the fibers). From the local form 16 of linear multivector fields, we see that these expressions determine $X$ completely. After a careful computation (needed to identify the brackets), one concludes that this construction defines an isomorphism of graded Lie algebras (note also that, since the map $X \mapsto D_{X}$ is local over $M$, one can work in local coordinates)

$$
\operatorname{Der}^{k-1}(E) \cong \mathcal{X}_{\text {lin }}^{k}\left(E^{\vee}\right)
$$

(This is a generalization to multivector fields of Proposition 2.4 of [15]; see also [12].) In particular, we deduce the following proposition, the first part of which is well-known (it first appeared in [2, Theorem 2.1.4]):

Proposition 7. Given a vector bundle A over $M$, there is a one-to-one correspondence between Lie algebroid structures on A and linear Poisson structures $\pi$ on $A^{\vee}$. Moreover, for any Lie algebroid A,

$$
H_{\mathrm{def}}^{*}(A) \cong H_{\pi, \operatorname{lin}}^{*}\left(A^{\vee}\right)
$$




\subsection{Rigidity}

Finally, we conjecture the following (cohomological) rigidity result similar to known rigidity results for compact Lie groups. The analogue for groupoids of the compactness property of groups is known as properness. For those aspects of Lie groupoids and properness which are relevant to our discussion, we refer to [3].

Conjecture 1. If $A$ is a Lie algebroid which admits a proper integrating groupoid $\mathcal{G}$ whose s-fibers are 2-simply connected, then $H_{\mathrm{def}}^{2}(A)=0$.

Such a result is relevant to the study of smooth deformations of Lie algebroids, and to linearization problems.

Proposition 8. The conjecture is true for regular algebroids, and for action algebroids.

Proof. First notice that by Theorem 4 and Proposition 1 of [3], the de Rham cohomology $H^{*}(A ; E)$ vanishes in degrees 1 and 2 , for any representation $E$. Now the regular case follows by Theorem 3 , and the action case by Proposition 5

We believe that the previous conjecture can be proven by further working in the lincategory (Subsection 4.9): one can talk about lin-algebroids (the LA-groupoids of Mackenzie [14]), lin-groupoids (the VB groupoids of Mackenzie [14]), etc., so that $H_{\mathrm{def}}^{*}(A)$ is isomorphic to the "lin-algebroid cohomology" of the lin-algebroid $T^{\vee} A^{\vee}$ (the algebroid associated to the lin-Poisson manifold $A^{\vee}$ ), and then try to prove a lin-version of the van Est-type results of [3] (for the lin-groupoid $T^{\vee} \mathcal{G}$ over $A^{\vee}$ ).

Acknowledgments. First drafts of this paper were written while the second author was visiting the University of Ljubljana (November 2000), and later while the second author was a Miller Fellow at UC Berkeley (2001). The main results of this paper were first presented during the Foliation Theory Program at the ESI in Vienna (October 2002). We are grateful to F. Kamber and K. Richardson for inviting us to this program, and to K. Mackenzie, J. Mrčun, A. Weinstein, and especially R. L. Fernandes, for helpful discussions related to this paper. After the paper was posted on the Archive, Y. Kosmann-Schwarzbach kindly pointed out several inaccuracies to us. In addition, we were informed that the (mere) definition of the deformation complex made its appearance in various guises in the work of several others, namely K. Grabowska et al. [9], D. Roytenberg (unpublished), A. Vaintrob [21] - we thank J. Grabowski, D. Roytenberg and the referee for these references.

The first author was partially supported by a KNAW Fellowship (Utrecht) and a Miller Fellowship (Berkeley).

\section{References}

[1] Conn, J. F.: Normal forms for smooth Poisson structures. Ann. of Math. 121, 565-593 (1985) Zbl 0592.58025 MR 0794374

[2] Courant, T. J.: Dirac manifolds. Trans. Amer. Math. Soc. 319, 631-661 (1990) Zbl 0850.70212 MR 0998124

[3] Crainic, M.: Differentiable and algebroid cohomology, Van Est isomorphisms, and characteristic classes. Comment. Math. Helv. 78, 681-721 (2003) Zbl 1041.58007| MR 2016690 
[4] Crainic, M., Fernandes, R. L.: Integrability of Lie brackets. Ann. of Math. 157, 575-620 (2003) Zbl 1037.22003 MR 1973056

[5] Evens, S., Lu, J. H., Weinstein, A.: Transverse measures, the modular class and a cohomology pairing for Lie algebroids. Quart. J. Math. Oxford Ser. (2) 50, 417-436 (1999) Zbl 0968.58014 MR 1726784

[6] Frölicher, A., Nijenhuis, A.: Theory of vector-valued differential forms. I. Derivations of the graded ring of differential forms. Nederl. Akad. Wetensch. Proc. Ser. A. 59 = Indag. Math. 18, 338-359 (1956) Zbl 0079.37502 MR 0082554

[7] Gerstenhaber, M.: The cohomology structure of an associative ring. Ann. of Math. 78, 267288 (1963) Zbl 0131.27302 MR 0161898

[8] Goldman, W. M., Millson, J. J.: The homotopy invariance of the Kuranishi space. Illinois J. Math. 34, 337-367 (1990) Zbl 0707.32004 MR 1046568

[9] Grabowska, K., Grabowski, J., Urbański, P.: Lie brackets on affine bundles. Ann. Global Anal. Geom. 24, 101-130 (2003) Zbl 1044.53056 MR 1990111

[10] Heitsch, J.: A cohomology for foliated manifolds. Comment. Math. Helv. 50, 197-218 (1975) Zbl 0311.57014 MR 0372877

[11] Kosmann-Schwarzbach, Y., Magri, F.: Poisson-Nijenhuis structures. Ann. Inst. H. Poincaré Phys. Théor. 53, 35-81 (1990) Zbl 0707.58048 MR 1077465

[12] Kosmann-Schwarzbach, Y.: Dérivées de Lie des morphismes de fibrés. In: Géométrie différentielle (Paris, 1976/1977), Univ. Paris VII, Paris, 55-71 (1978) MR 0687085

[13] Mackenzie, K.: Lie Groupoids and Lie Algebroids in Differential Geometry. London Math. Soc. Lecture Note Ser. 124, Cambridge Univ. Press (1987) Zbl 0683.53029 MR 0896907

[14] Mackenzie, K.: Double Lie algebroids and second-order geometry. Adv. Math. 94, 180-239 (1992) Zbl 0765.57025 MR 1174393

[15] Mackenzie, K., Xu, P.: Classical lifting processes and multiplicative vector fields. Quart. J. Math. Oxford Ser. (2) 49, 59-85 (1998) Zbl 0926.58015 MR 1617335

[16] Moerdijk, I., Mrčun, J.: On integrability of infinitesimal actions. Amer. J. Math. 124, 567-593 (2002) Zbl 1013.58010 MR 1902889

[17] Nijenhuis, A., Richardson, R. W.: Cohomology and deformations in graded Lie algebras. Bull. Amer. Math. Soc. 72, 1-29 (1966) Zbl 0136.30502 MR 0195995

[18] Nijenhuis, A., Richardson, R. W.: Deformations of homomorphisms of Lie groups and Lie algebras. Bull. Amer. Math. Soc. 73, 175-179 (1967) Zbl 0153.04402 MR 0204575

[19] Nijenhuis, A., Richardson, R. W.: Commutative algebra cohomology and deformations of Lie and associative algebras. J. Algebra 9, 42-53 (1968) Zbl 0175.31302 MR 0225830

[20] Roytenberg, D.: On the structure of graded symplectic supermanifolds and Courant algebroids. In: Quantization, Poisson Brackets and Beyond (Manchester, 2001), Contemp. Math. 315, Amer. Math. Soc., Providence, RI, 169-185 (2002) Zbl 1036.53057 MR 1958835

[21] Vaintrob, A.: Lie algebroids and homological vector fields. Russian Math. Surveys 52, 428429 (1997) Zbl 0955.58017 MR 1480150

[22] Vaisman, I.: Lectures on the Geometry of Poisson Manifolds. Progr. Math. 118, Birkhäuser, Berlin (1994) Zbl 0810.53019 MR 1269545

[23] Weinstein, A.: Linearization problems for Lie algebroids and Lie groupoids. In: Conference Moshé Flato 1999 (Dijon, 1990), Lett. Math. Phys. 52, 93-102 (2000) Zbl 0961.22004 MR 1800493

[24] Weinstein, A.: Linearization of regular proper groupoids. J. Inst. Math. Jussieu 1, 493-511 (2002) Zbl 1043.58009 MR 1956059

[25] Xu, P.: Gerstenhaber algebras and BV-algebras in Poisson geometry. Comm. Math. Phys. 200, 545-560 (1999) Zbl 0941.17016 MR 1675117 